\title{
Microstructure and mechanical properties of unsaturated polyester composites filled with waste rubber glove crumbs
}

\begin{abstract}
Recycling of waste rubber gloves is an alternative to sustainable methods in ensuring that waste rubber does not pile up. The main focus of this investigation is to evaluate the potential of utilising waste rubber gloves as fillers in unsaturated polyester-based composites. The evaluation also studies the effect of different loading of rubber content towards the composite's mechanical properties with 8 different rubber contents being considered. Rubber contents of $5,10,15,20,25,30,35$ and $40 \mathrm{wt}$. \% have been studied in this work. Waste rubber gloves were ground into crumbs using cryogenic grinding and added into the unsaturated polyester. The composite is characterised for its tensile and flexural strength, elastic modulus, impact strength and fracture surface morphology. The addition of rubber crumbs has increased the toughness of the composite while it decreased the tensile strength, flexural strength and elastic modulus. Due to rubber hydrophobic behaviour, adhesion between rubber crumbs and polyester gets weaker as the rubber content was increased, therefore the tensile strength of the composite dropped. The SEM micrographs of the tensile fracture surface of the composite revealed that voids in the composite increases as the rubber content is increased. Voids occur due to inhomogeneous distribution of rubber crumbs throughout the composite
\end{abstract}

Keyword: Waste rubber glove; Rubber crumbs; Unsaturated polyester composite; Mechanical properties 